\title{
Immunotherapy with toripalimab for lung adenocarcinoma in a real-world patient with an Eastern Cooperative Oncology Group performance status (ECOG PS) score of 4: a case report
}

\author{
Yanfeng Xue ${ }^{1}$, Kang Zheng ${ }^{1}$, Jinmei Xue ${ }^{2}$ \\ ${ }^{1}$ General VIP Ward, Shanxi Cancer Hospital, Taiyuan, China; ${ }^{2}$ Department of Otolaryngology, Second Hospital of Shanxi Medical University, \\ Taiyuan, China \\ Correspondence to: Jinmei Xue. Department of Otolaryngology, Second Hospital of Shanxi Medical University, Taiyuan, China. Email: xjment@126.com.
}

\begin{abstract}
Multidisciplinary treatment, mainly chemotherapy combined with immunotherapy, is preferred in patients with advanced lung cancer who are negative for driver genes and have an Eastern Cooperative Oncology Group performance status (ECOG PS) score of $0-1$; in contrast, patients with an ECOG PS score of 3-4 should be managed with supportive treatment and palliative care rather than chemotherapy or other antitumor treatments. In the real-world settings, however, in the Chinese population, a large proportion of patients and their families are willing to take risks to receive benefit from oncological treatments. We encountered a patient who had definite advanced lung adenocarcinoma with multiple metastases and fusion in abdominal lymph nodes. Intestinal obstruction and obstructive jaundice were also observed. Despite the application of gastrointestinal decompression, continuous parenteral nutrition support, common bile duct stenting, and pancreatic duct stenting, no effective antitumor therapy (except in the case of immunotherapy) was attempted due to the high tumor burden and poor ECOG PS score in this patient. After effective communication with the family members, toripalimab was attempted in this patient with great success (the patient did not receive chemotherapy and only received immunotherapy). In the spring of 2019, imported programmed death receptor-1 (PD-1) antibodies (pabolizumab and navulizumab) listed in the Chinese market were expensive, and the people could not afford the cost. However, treprizumab was just on the market, with low price and high pharmacoeconomics. Therefore, the drug was selected for treatment.) The patient achieved almost complete remission, with a progression-free survival (PFS) of about 2.5 years. The expression of programmed death-ligand 1 (PD-L1) was high in this patient. Toripalimab monotherapy may be applied in patients with high PD-L1 expression although any potential adverse effects should be closely monitored. To our knowledge, no other data from clinical trials and real-world cases have thus far been reported concerning the application of immunotherapy in ECOG PS 4 patients with advanced lung cancer. The innovation of this article is that although the patient has advanced cancer and ECOG PS 4 is an endstage patient, immunotherapy is still given and achieved great success.
\end{abstract}

Keywords: Real-world; Eastern Cooperative Oncology Group performance status 4 (ECOG PS 4); lung adenocarcinoma; immunotherapy; case report

Submitted Jul 26, 2021. Accepted for publication Sep 18, 2021.

doi: 10.21037/apm-21-2548

View this article at: https://dx.doi.org/10.21037/apm-21-2548

\section{Introduction}

In the last decade, the introduction of immune checkpoint inhibitors (ICIs) has dramatically improved the prognosis of patients with non-small cell lung cancer (NSCLC). First- line ICI or chemo-ICI trials have demonstrated benefits to overall survival (OS) but mainly for those patients with an Eastern Cooperative Oncology Group performance status (ECOG PS) from 0 to 1 . In the real world, there is a 
greater proportion patients with an ECOG PS score from 3 to 4 , for whom palliative care and nutritional support are recommended in the currently available guidelines.

We encountered a patient with lung cancer patient and an ECOG PS score of 4 . His PS score was already poor ( $\mathrm{PS}=2$ ) at admission, which worsened during the wait for pathological results. Despite active communication with the patient's family members, the latter still desired aggressive antitumor therapy for the patient. ICI monotherapy was proposed, but the price of pablizumab was high in China at that time and thus not acceptable to the patient's family. Instead, toripalimab (Shanghai Junshi Bioscience Co., Ltd., Shanghai, China) which was affordable for them, was agreed upon. As a recombinant human anti-programmed death receptor-1 (anti-PD-1) monoclonal antibody, toripalimab, is the first China-developed PD-1 inhibitor. Its effects work according to a similar therapeutic principle as that of pablizumab. Our current patient received 35 sessions of toripalimab treatment, with excellent response. We present the following article in accordance with the CARE reporting checklist (available at https://dx.doi.org/10.21037/ apm-21-2548).

\section{Case presentation}

In July 2018, a 65-year-old male was experiencing wasting, fatigue, and night sweats without any obvious causes but had no fever, nausea, vomiting, abdominal pain, diarrhea, or other discomforts. No special treatment was given. As these symptoms and signs persisted, he visited a local hospital in December 2019 and received complete examinations. Color Doppler ultrasound showed multiple swollen lymph nodes in both sides of his neck. Computed tomography (CT) showed multiple enlarged lymph nodes in the mediastinum and multiple enlarged lymph nodes in the abdominal cavity and retroperitoneum. Retroperitoneal lymph node biopsy was then performed in that hospital. Our Shanxi Cancer hospital was consulted for the postoperative pathology, which showed the presence of atypical cells, and the possibility of a malignancy could not be ruled out; as there was a very small amount of tissue available for diagnosis, complete lymph node dissection (CLND) or immunohistochemistry (IHC) was recommended for further diagnosis. The patient was admitted to the hematology department of our hospital on March 15, 2019. Before admission, he had coronary artery disease for more than 1 year and underwent cardiac vascular stenting more than 1 year prior. He was orally administered with atorvastatin, trimetazidine tablets, hydrochlorothiazide tablets, and isosorbide mononitrate tablets. His heart disease was stable, and there were no angina symptoms. He denied having a medical history for any other conditions. He also denied tobacco use or alcohol/recreational drug abuse. He had no family history of tumor.

\section{Examination results at admission}

The CT mediastinal window showed multiple enlarged lymph nodes in both clavicular regions, and in the mediastinum, mesentery, retroperitoneum, and right iliac vessels. The possibility of malignancy could not be ruled out, and further detailed examinations were recommended. Mild intrahepatic ductal dilatation was observed, and effusions were observed in the abdominopelvic, pericardial, and bilateral pleural areas.

Images obtained with lung window settings showed multiple small nodules in the upper and middle lobes of both lungs, multiple inflammatory lesions in the lingual segment of the upper lobe of the left lung and the lower lobe of both lungs, and calcified foci in the lingual segment of the upper lobe of the left lung. Further examinations showed the swelling of the group IV lymph nodes in left neck. Lymph node puncture and pathology confirmed the presence of metastatic carcinoma.

The immunohistochemical results were as follows: AE1/AE3 (+), P63 (-), P40 (-), CAM5.2 (+), CK20 (-), Ki-67 (about 40\%+), Syn (-), and Cga (-). These results were consistent with the diagnosis of a metastatic adenocarcinoma. The possibility of blood malignancy was excluded. The patient was then transferred to our department on the 13th day of admission for further treatment.

\section{Clinical manifestations at transfer}

At the time of transfer, the patient was in poor general condition: his mental status and appetite were poor; abdominal distension, nausea, and vomiting were obvious; and there was a small amount of urine and stool. Physical examination showed his skin and sclera were yellowish, and enlarged lymph nodes of different sizes could be palpated in both sides of the necks and near both clavicles; breath sounds in both lungs were clear, and there were no dry rales; his abdomen was distended and positive for mobile turbid sounds, and both lower limbs showed mild swelling. His ECOG PS score was 4. 


\section{Treatments after transfer}

The following procedures were completed after transfer: (I) further pathology and IHC were performed to search for the source of the primary lesion. (II) Peritoneal fluid puncture for catheter placement was immediately performed for intermittent discharge of ascites and decompression therapy. (III) A central catheter (PICC) was peripherally inserted, and parenteral nutrition therapy was administered. Laboratory tests were also conducted to identify the cause of jaundice. (IV) Gastroscopy and enteroscopy were performed, which showed no significant abnormalities.

After hepatoprotection and nutritional support, liver and kidney function tests were performed on March 28, 2019, and April 1, 2019, respectively: total bilirubin was found to be progressively elevated, while direct bilirubin was significantly increased. Obstructive jaundice was considered. Based on the CT findings, jaundice was speculated to be caused by the enlarged lymph nodes around the head of the pancreas and at the mesenteric root, which fused and thus compressed the duodenal papilla. Abdominal distension was not alleviated after the discharge of ascites; rather, it became worse and was accompanied by nausea and vomiting. Accordingly, indwelling gastric tube placement and gastrointestinal decompression were performed on April 2, 2019, and endoscopic metal stenting into the bile duct and endoscopic plastic stenting into the pancreatic duct were conducted on April 3, 2019. The symptoms of obstructive jaundice were significantly improved after these treatments.

On April 4, 2019, IHC showed TTF-1 (+), Napsin-a (+), and CDX-2 (-), suggesting a high possibility of a lung origin of the lesion. Lung cancer-related gene testing revealed no relevant gene mutation. Unfortunately, programmed deathligand 1 (PD-L1) expression testing was not being carried out in our hospital at that time, and thus the PD-L1 level remained unknown.

The diagnoses made were as follows: metastatic adenocarcinoma in the left cervical lymph nodes (possible lung origin); multiple lymph node metastases in both clavicular areas, and in the mediastinum, mesentery, retroperitoneum, and right iliac vessels; obstructive jaundice; small bowel obstruction; peritoneal effusion; bilateral pleural effusions; pericardial effusion; coronary atherosclerosis; tumor-node-metastasis (TNM) status of $\mathrm{T}_{\mathrm{x}} \mathrm{N}_{3} \mathrm{M}_{1}$; stage IV disease; and an ECOG PS score of 4. A summary of his medical features is as follows: male, 65 years old, stage IV lung cancer, huge tumor burden, obstructive jaundice with poor liver function, gastrointestinal obstruction with parenteral nutrition support being offered, and hypoproteinemia. His ECOG PS score was 4 points. Both the National Comprehensive Cancer Network (NCNN) guidelines and the 2019 Clinical Study Oversight Committee (CSOC) guidelines recommend that ECOG PS 4 patients be treated with supportive care; however, the patient's family had a strong desire to treat the tumor.

According to the results of literature review, the optimal treatment for ECOG PS 4 patients is supportive care; no relevant case report has been published in Chinese or English journals, and no article has described the application of chemotherapy and immunotherapy for advanced lung cancer patients with poor ECOG PS scores. Thus, adhering to the family's wishes was a difficult issue for us to consider.

Based on the request and the financial means of the patient's family, $240 \mathrm{mg}$ of intravenous toripalimab treatment was initiated on April 4, 2019, and repeated every 3 weeks. The treatment was planned to last 2 years if it was effective.

With the use of toripalimab, the patient's condition gradually improved, as evidenced by the gradual reduction of ascites and the gradual improvement of abdominal distension. The parenteral nutrition status was gradually progressed to drinking water, clear liquid food, semiliquid diet, and a normal diet. The patient revisited our hospital after completing 4 cycles of chemotherapy, and he had good mental status and appetite and could eat normally. The abdominal drainage tube was removed, and his body weight increased by $5 \mathrm{~kg}$. He received intermittent examinations and monitoring in the coming months, as shown in Figures 1,2.

All the steps in this study involving human participants were in accordance with the ethical standards released by the institutional and/or national research boards and the Declaration of Helsinki (as revised in 2013). Written informed consent was obtained from the patient for publication of this case report and accompanying images. A copy of the written consent is available for review by the editorial office of this journal.

Due to the COVID-19 outbreak in China at the end of January 2020, the patient was followed up via telephone, during which any adverse effects were queried and the treatment response was evaluated based on symptoms. The patient received immunotherapy at a clinic near his home or at a community hospital. As the COVID-19 outbreak improved, he received another CT scan in a local hospital on December 11, 2020, which showed that he had stable disease (Figure 3). 

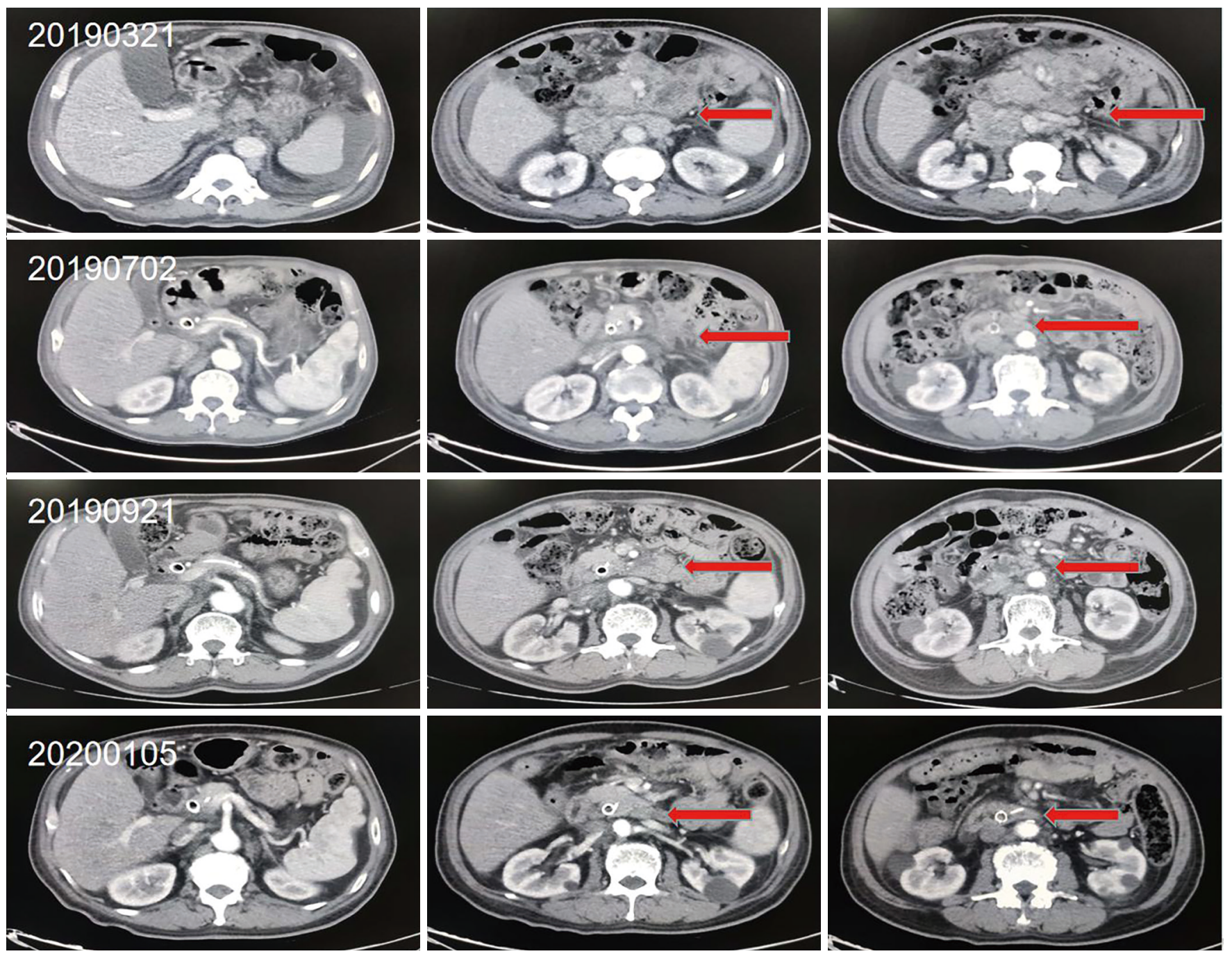

Figure 1 Mediastinal window images from disease onset (March 2019) to treatment sessions (January 2020). The content indicated by the arrow: it is the state of lymph node metastasis, enlargement and fusion, which is to emphasize.

On May 4, 2021, the patient received examinations again in another hospital, which demonstrated that he had achieved complete remission. Meanwhile, as toripalimab had been administered for 2 years, it was withdrawn in that month (Figure 4).

No adverse reactions or side effects (immune-related rash, immune-related pneumonia, etc.) were noted throughout the 2-year treatment. On December 16, 2020, we obtained the patient's initial puncture results, and subsequent $\mathrm{PD}-\mathrm{L} 1$ testing and microsatellite instability (MSI) testing showed high expression of PD-L1, [PD-L1 tumor proportion score (TPS): 70\%] and MSI-low (MSI-L).

\section{Discussion}

In order to conduct clinical trials of cancer treatment in the numerous participating hospitals, cancer centers, and clinics in a consistent manner, it is necessary to use uniform practices to measure how the disease affects the patient's ability of daily living (i.e., the patient's performance state). The ECOG PS is such a measure. It describes the patient's functional level according to the patient's self-care ability, daily activities, and physical ability (walking, work, etc.). Researchers around the world consider ECOG PS to study a new treatment when planning trials. The scale is a method to define the group of patients to be studied in the trial, 

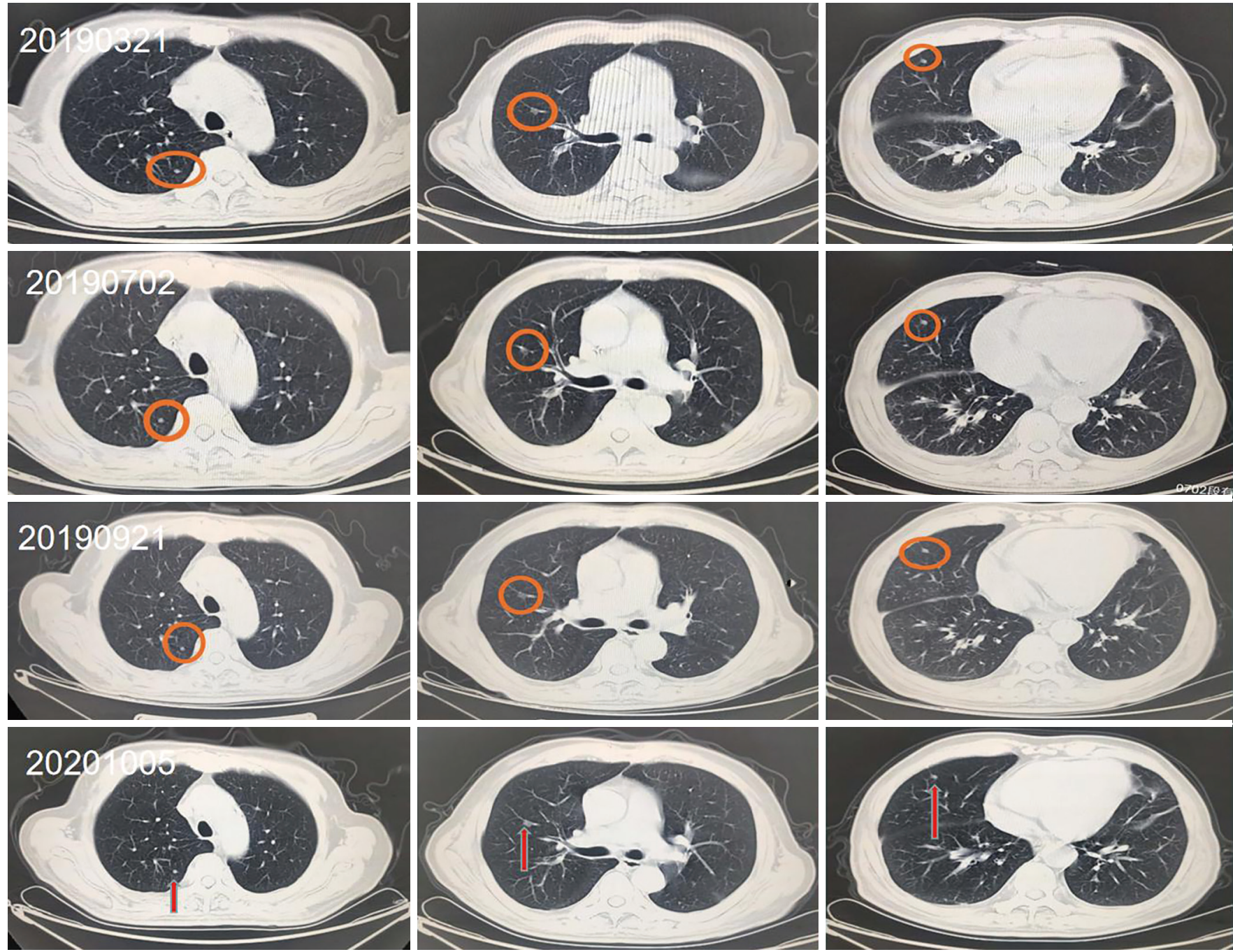

Figure 2 Mediastinal window images from disease onset (March 2019) to treatment sessions (January 2020). The red circle shows that there are only a few pulmonary nodules and no large pulmonary masses at the beginning of the disease on the CT lung window. With each reexamination, all metastases were reduced, but there was no significant change in pulmonary nodules. Red circles and red arrows were marked for highlighting and emphasis.

so that doctors who recruit patients can uniformly copy the scale. This is also a way for doctors to track changes in patients' functional levels through treatment during the trial. First published in 1982, the scale was developed by ECOG, which is now part of the ECOG-ACRIN (American College of Radiology Imaging Network) Cancer Research Group (1). The definition of PS 4 is total disability, inability to perform any self-care, and complete confinement to a bed or chair.

The American Society of Clinical Oncology (ASCO) guidelines recommend that chemotherapy should not be used in patients with solid tumors who have not previously received treatment and have an ECOG PS of 3 or more (i.e., only limited self-care, lying in bed or sitting in a chair for more than $50 \%$ of their waking time). This suggestion was supported by a study in the 1980s, which found that patients with low PS score had low chemotherapy response rate (RR), high incidence of toxicity and short survival (2). As patients with a good PS are expected to benefit most from chemotherapy, trials targeting these patients have been conducted and have largely excluded cancer patients with poor PS $(3,4)$. Before the era of immunotherapy, 

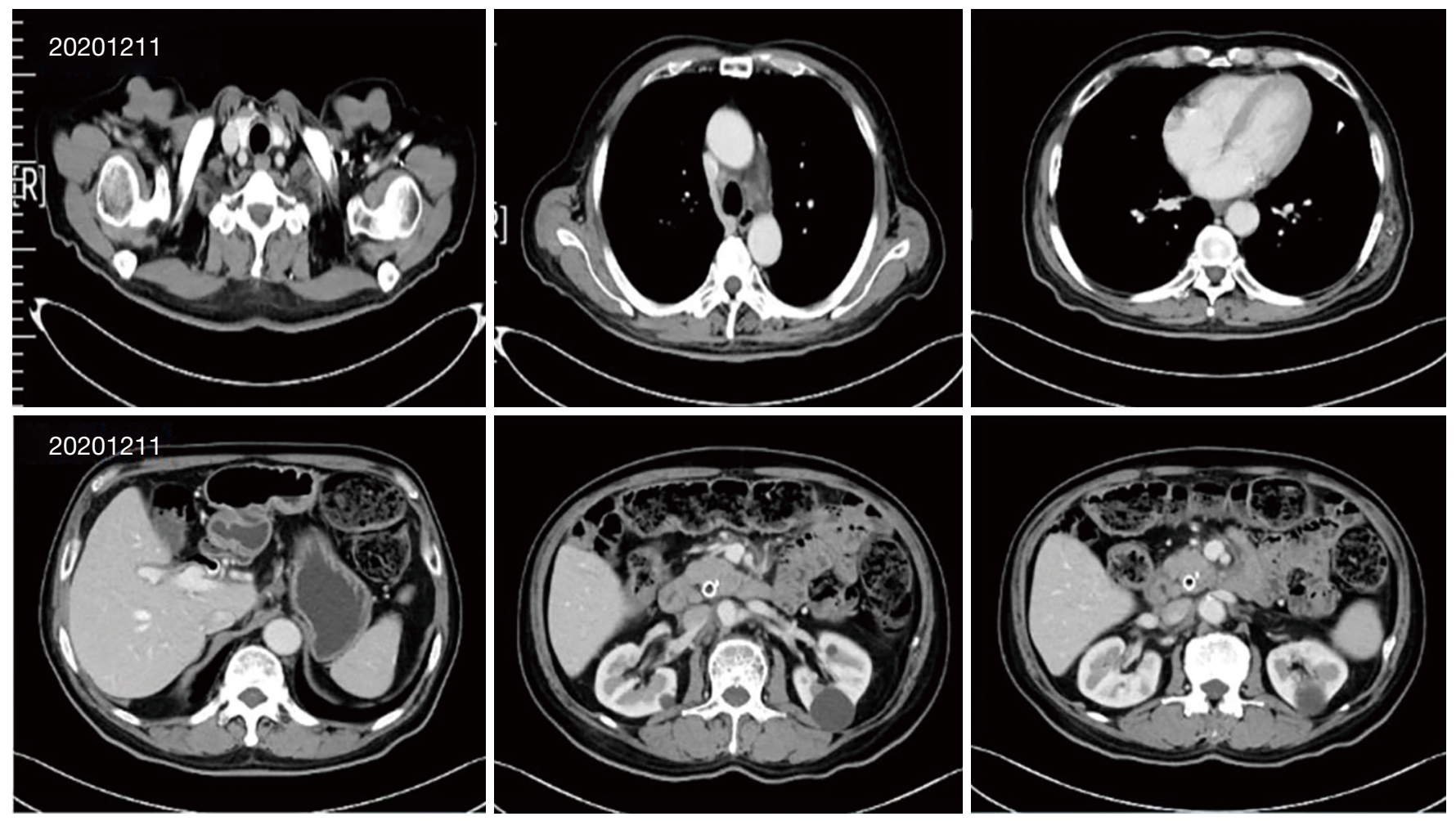

Figure 3 Disease status on December 11, 2020.

clinical trials evaluating chemotherapy in patients with NSCLC were divided into patients with PS $0-1$ and PS 2 (5). These clinical trials showed that PS is the most powerful independent predictor of chemotherapy response in patients with advanced NSCLC.

In the past decade, the introduction of ICIs has significantly improved the prognosis of patients with NSCLC. First line ICI or chemotherapy ICI trials have been shown to be beneficial to OS, but only in patients with ECOG PS of 0 or 1 .

The vast majority of ICI studies have excluded patients with poor PS (>2). PS 2 is especially considered to be a negative prognostic factor for survival and a predictor of adverse events and adverse treatment reactions. Data on ICIs activity in PS 2 patients are limited and come from heterogeneous meta-analysis and small phase II or extended access trials. Generally, the terms "unhealthy" or "weak" describe the eligibility of patients to receive cytotoxic chemotherapy, without specifying PS (6).

A review of the literature shows that only a small number of randomized controlled clinical trials have enrolled patients with PS 2 and above. CheckMate 171 is a phase 2 trial of nivolumab in previously treated advanced squamous
NSCLC patients (including ECOG PS 2 patients and older adults) conducted as part of a postapproval commitment to the European Medicines Agency (EMA). Of 811 patients treated, 103 had ECOG PS 2; 278 were aged $\geq 70$ years, and 125 were $\geq 75$ years of age. The minimum follow-up was about 18 months. Nivolumab was well tolerated and the clinical profile of nivolumab was similar between the older adults and the general population. However, PS2 patients had a significantly shorter OS (5.4 vs. 9.9 months) compared to the general population (PS 0-1), but there was no significant difference in safety profiles between these two groups (7).

In a single-arm phase 2 trial (PePS2) on pembrolizumab for NSCLC in PS2 patients, 60 patients with NSCLC and a rigorous ascription of PS2 were given $200 \mathrm{mg}$ of pembrolizumab every 3 weeks. The treatment evaluation was stratified by TPS and line of therapy. The observed incidence for durable clinical benefit (DCB) was 38\% [95\% confidence interval (CI): $21-57 \%$ ] in first-line patients $(n=24)$ and $36 \%(95 \%$ CI: $22-52 \%)$ in subsequent-line patients $(\mathrm{n}=36)$; DCB was $22 \%$ (95\% CI: $11-41 \%)$ in patients with a TPS less than $1 \%(\mathrm{n}=27), 47 \%(95 \%$ CI: $25-70 \%)$ in patients with a TPS of $1-49 \%(n=15)$, and 

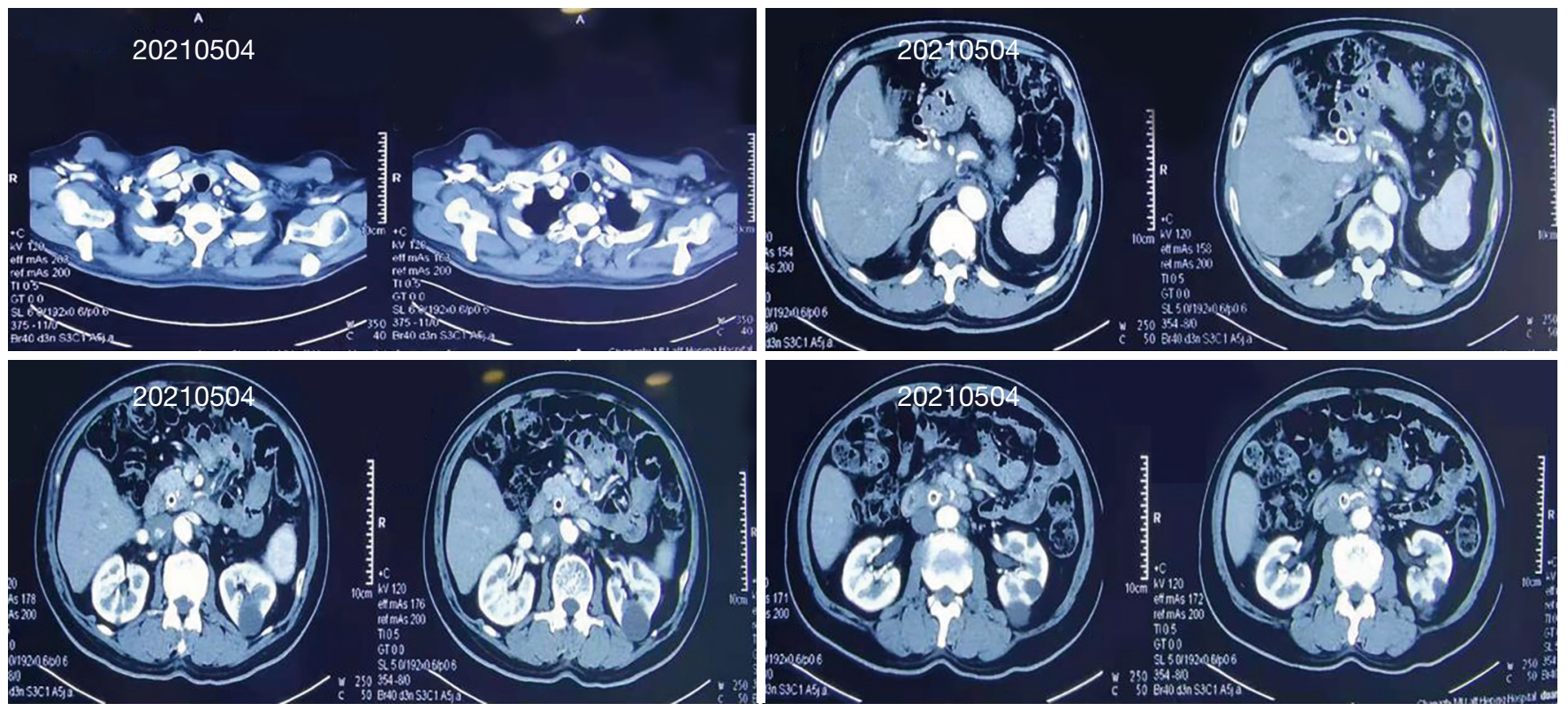

Figure 4 Treatment response after 2 years of toripalimab use.

$53 \%$ (95\% CI: $30-75 \%$ ) in patients with a TPS of $50 \%$ or greater $(n=15)$. No grade 5 treatment-related adverse events were observed, and no early deaths were attributable to hyperprogression. Thus, the results of this trial were quite encouraging (8).

In fact, quite a few retrospective studies have enrolled patients with poor ECOG PS. Adachi et al. retrospectively collected pretreatment clinical data of 296 consecutive patients with NSCLC treated with nivolumab. The aim of this retrospective study was to determine the predictors of nivolumab response in patients with NSCLC with good PS (PS 0-1) and poor PS (PS 2-4). Notably, PS 4 patients were included in this analysis. PS was found to be associated with progression-free survival (PFS) in patients with NSCLC treated with nivolumab. The predictors of nivolumab response were different between NSCLC patients with good PS and NSCLC patients with poor PS. Never smoking status, high C-reactive protein (CRP), liver metastasis, pleural effusion, use of steroids at the beginning of nivolumab treatment predicted poor PFS in NSCLC patients with good PS, while high advanced pulmonary inflammation index (ALI) predicted better PFS in patients with poor PS. Furthermore, 6 of 296 patients had PS 4, and all of them had progressive disease (PD) after treatment. However, PD-L1 expression status could not be assessed as a predictive factor because of the lack of routine PD-L1 testing (9).
Immunization with pablizumab has been applied in patients with a PD-L1 TPS of more than $50 \%$. Although immunotherapy and chemoimmunotherapy have changed the current treatment paradigm, most published studies have only enrolled medically eligible patients (10-16).

PS is the most powerful independent prognostic factor in advanced NSCLC and a predictor of survival and adverse events (17). When receiving conventional chemotherapy, patients with PS 2 and PS 3 have a worse prognosis and higher toxicity rates, which is why they are excluded from many clinical trials $(18,19)$. For patients with PS $0-1$, standard treatment is usually recommended, while for patients with PS 4, the best supportive treatment is provided. Clinical trials are needed to determine the best practices for PS 2 and PS 3 patients. In a previous study, PS 2 and PS 3 patients accounted for approximately $30 \%$ and $15 \%$ of lung cancer patients, respectively. These proportions may not adequately represent the full population in randomized trials (20). Given the toxicity profile of immunotherapy, it is unclear whether the ECOG scale will continue to have predictive value when using these kinds of new agents. In a retrospective analysis performed in 2020, 285 NSCLC patients who received immunotherapy (mainly nivolumab, pablizumab, atezolizumab, or ipilimumab) between January 2014 and April 2018 were identified. In this group, 153 patients (53.7\%) had PS 0-1, 114 (40.0\%) had PS 2, and $18(6.3 \%)$ had PS 3. RRs were similar across PS groups, 
with $26.6 \%$ for PS $1,25.2 \%$ for PS 2 , and $23.1 \%$ for PS 3 $(\mathrm{P}=0.95)$. Survival outcomes varied with pretreatment PS. For PS 0-1, PS 2, and PS 3, the median OS was 14.7, 8.3, and 1.5 months $(\mathrm{P}<0.001)$, and the PDS was 7.4 , 5.1, and 1.3 months $(\mathrm{P}<0.001)$. The authors concluded that patients with poor baseline PS showed similar RR compared with healthy patients, but PFS and OS were poor (21).

In summary, only a few PS 2 patients have generally been enrolled in randomized controlled trials that can be tracked in the open platforms, with most of the related findings showing that the RR of immunotherapy is low in PS 2 patients and that the PSF and OS in PS 2 patients are shorter than those of PS $0-1$ patients. Although PS 3-4 patients have also been enrolled in some retrospective pilot studies, the RRs have been low and the survival short. However, in our current study, the patient had a PS score of 4, along with high tumor burden, obstructive jaundice, and intestinal obstruction. His disease condition was alleviated after only 1 cycle of monotherapy with toripalimab, and the patient reached an ECOG PS score of 0 after 4 cycles of treatment. He can now eat normally and participate in work.

Toripalimab (Shanghai Junshi Bioscience Co., Ltd., Shanghai, China) is the first PD-1 inhibitor to be developed exclusively in China. The related clinical trial showed that, in patients with unresectable or metastatic melanoma who had failed in prior systemic therapy, the objective RR was $17.3 \%$, the disease control rate was $57.5 \%$, and the 1-year survival rate was $69.3 \%$. The clinical research and development of toripalimab began in early 2016, and 30 clinical trials covering 14 tumor types have been conducted thus far in many countries including China and the United States. In an original article published by Wang et al. from the Cancer Hospital, Chinese Academy of Medical Sciences and Peking Union Medical College, in $7 A M A$ Network Open on October 5, 2020, the authors assessed the safety, antitumor activity, and pharmacokinetics of toripalimab in patients with advanced NSCLC and evaluated the utility of JS311, a novel PD-L1 IHC assay. As shown by the JS311 assay, good long-term survival and durable response were achieved in patients with PD-L1 expression greater than $50 \%$ (22). Nevertheless, toripalimab was not approved for treating lung cancer in China in March 2019, and there are no reliable data concerning its efficacy in treating lung cancer.

In the case presented here, toripalimab treatment was started after informed consent from the family members and notification of their strong desire for treatment had been received. When the patient applied immune drugs for 2 years, the patient was rechecked. The patient's condition was stable, so he was stopped for observation and followed up for half a year (Figure 4). As of this writing, the PFS has reached 2.5 years and the patient remains alive. He has good mental status and appetite, and his body weight has increased by $10 \mathrm{~kg}$. His current ECOG PS score is 0 , and the patient has not experienced any immune-related adverse reactions throughout the treatment. The breakthough success of toripalimab monotherapy in this patient raises an important question: is toripalimab highly efficacious in patients with high PD-L1 expression, even if their ECOG PS scores are poor? As our data were derived from just a single patient, data from a greater number of cases are needed to further explore this question.

Many patients with lung adenocarcinoma with ECOG PS 4 can be seen clinically. Generally speaking, these patients have multiple metastases, large tumor load, high nutritional risk, high infection risk and short survival time. Generally, they are not given active treatment, but only palliative nutritional support and pain relief. However, some families are willing to try anti-tumor treatment (for example, palliative chemotherapy while full nutrition, for example, patients with high expression of PD-L1 can try to apply PD-1 antibody). In the real world, the premise of all this must be the active cooperation and treatment of patients and their families, and they are willing to take risks, because patients with PS score of 4 are at the end of clinical stage, and active anti-tumor treatment is no longer recommended in the guidelines. But at the same time, thank patients and their families who have the spirit of risk, if not them Adhering to immunotherapy, patients can not achieve great success. Perhaps poor PS score should not be a contraindication of immunotherapy.

\section{Acknowledgments}

Funding: None.

\section{Footnote}

Reporting Checklist: The authors have completed the CARE reporting checklist. Available at https://dx.doi. org/10.21037/apm-21-2548

Conflicts of Interest: All authors have completed the ICMJE uniform disclosure form (available at https://dx.doi. org/10.21037/apm-21-2548). The authors have no conflicts 
of interest to declare.

Ethical Statement: The authors are accountable for all aspects of the work in ensuring that questions related to the accuracy or integrity of any part of the work are appropriately investigated and resolved. All the steps in this study involving human participants were in accordance with the ethical standards released by the institutional and/or national research boards and the Declaration of Helsinki (as revised in 2013). Written informed consent was obtained from the patient for publication of this case report and accompanying images. A copy of the written consent is available for review by the editorial office of this journal.

Open Access Statement: This is an Open Access article distributed in accordance with the Creative Commons Attribution-NonCommercial-NoDerivs 4.0 International License (CC BY-NC-ND 4.0), which permits the noncommercial replication and distribution of the article with the strict proviso that no changes or edits are made and the original work is properly cited (including links to both the formal publication through the relevant DOI and the license). See: https://creativecommons.org/licenses/by-nc-nd/4.0/.

\section{References}

1. Eastern Cooperative Oncology Group. ECOG performance status. [Acessed June 3, 2015]. Available online: http://ecog-acrin.org/resources/ecog-performancestatus

2. Schnipper LE, Smith TJ, Raghavan D, et al. American Society of Clinical Oncology identifies five key opportunities to improve care and reduce costs: the top five list for oncology. J Clin Oncol 2012;30:1715-24.

3. Stanley KE. Prognostic factors for survival in patients with inoperable lung cancer. J Natl Cancer Inst 1980;65:25-32.

4. Pater JL, Loeb M. Nonanatomic prognostic factors in carcinoma of the lung: a multivariate analysis. Cancer 1982;50:326-31.

5. Schiller JH, Harrington D, Belani CP, et al. Comparison of four chemotherapy regimens for advanced non-smallcell lung cancer. N Engl J Med 2002;346:92-8.

6. Friedlaender A, Banna GL, Buffoni L, et al. Poorperformance status assessment of patients with nonsmall cell lung cancer remains vague and blurred in the immunotherapy era. Curr Oncol Rep 2019;21:107.

7. Felip E, Ardizzoni A, Ciuleanu T, et al. CheckMate 171: A phase 2 trial of nivolumab in patients with previously treated advanced squamous non-small cell lung cancer, including ECOG PS 2 and elderly populations. Eur J Cancer 2020;127:160-72.

8. Middleton G, Brock K, Savage J, et al. Pembrolizumab in patients with non-small-cell lung cancer of performance status 2 (PePS2): a single arm, phase 2 trial. Lancet Respir Med 2020;8:895-904.

9. Adachi Y, Tamiya A, Taniguchi $Y$, et al. Predictive factors for progression-free survival in non-small cell lung cancer patients receiving nivolumab based on performance status. Cancer Med 2020;9:1383-91.

10. Reck M, Rodríguez-Abreu D, Robinson AG, et al. Pembrolizumab versus chemotherapy for PD-L1positive non-small-cell lung cancer. $\mathrm{N}$ Engl J Med 2016;375:1823-33.

11. Gandhi L, Rodríguez-Abreu D, Gadgeel S, et al. Pembrolizumab plus chemotherapy in metastatic nonsmall-cell lung cancer. N Engl J Med 2018;378:2078-92.

12. Paz-Ares L, Luft A, Vicente D, et al. Pembrolizumab plus chemotherapy for squamous non-small-cell lung cancer. $\mathrm{N}$ Engl J Med 2018;379:2040-51.

13. Socinski MA, Jotte RM, Cappuzzo F, et al. Atezolizumab for first-line treatment of metastatic nonsquamous NSCLC. N Engl J Med 2018;378:2288-301.

14. Borghaei H, Paz-Ares L, Horn L, et al. Nivolumab versus docetaxel in advanced nonsquamous non-small-cell lung cancer. N Engl J Med 2015;373:1627-39.

15. Herbst RS, Baas P, Kim DW, et al. Pembrolizumab versus docetaxel for previously treated, PD-L1-positive, advanced non-small-cell lung cancer (KEYNOTE-010): a randomised controlled trial. Lancet 2016;387:1540-50.

16. Brahmer J, Reckamp KL, Baas $\mathrm{P}$, et al. Nivolumab versus docetaxel in advanced squamous-cell non-small-cell lung cancer. N Engl J Med 2015;373:123-35.

17. Sculier JP, Chansky K, Crowley JJ, et al. The impact of additional prognostic factors on survival and their relationship with the anatomical extent of disease expressed by the 6th Edition of the TNM Classification of Malignant Tumors and the proposals for the 7th Edition. J Thorac Oncol 2008;3:457-66.

18. Bunn PA Jr. Chemotherapy for advanced non-smallcell lung cancer: who, what, when, why? J Clin Oncol 2002;20:23S-33S.

19. Tartarone A, Aieta M. Treatment of performance status 2 patients with advanced non-small-cell lung cancer: what we know and what we don't know. Future Oncol 2009;5:837-41.

20. Lilenbaum RC, Cashy J, Hensing TA, et al. Prevalence 
of poor performance status in lung cancer patients: implications for research. J Thorac Oncol 2008;3:125-9.

21. Ahmed T, Lycan T, Dothard A, et al. Performance status and age as predictors of immunotherapy outcomes in advanced non-small-cell lung cancer. Clin Lung Cancer 2020;21:e286-93.

22. Wang Z, Ying J, Xu J, et al. Safety, antitumor activity,

Cite this article as: Xue Y, Zheng K, Xue J. Immunotherapy with toripalimab for lung adenocarcinoma in a real-world patient with an Eastern Cooperative Oncology Group performance status (ECOG PS) score of 4: a case report. Ann Palliat Med 2021;10(9):10114-10123. doi: 10.21037/apm-21-2548 and pharmacokinetics of toripalimab, a programmed cell death 1 inhibitor, in patients with advanced nonsmall cell lung cancer: a phase 1 trial. JAMA Netw Open 2020;3:e2013770.

(English Language Editor: J. Gray) 